\title{
Desarrollo del pensamiento crítico: Un reto para profesores de literatura
}

\author{
Lourdes Gómez Precilla ${ }^{1}$ \\ Universidad de Panamá \\ teacherlgg@yahoo.com
}

\section{Resumen}

Los seres humanos poseen conocimientos que van adquiriendo a lo largo de su vida. Sin embargo, esos saberes pueden desarrollarse de forma diferente por los modelos y enfoques que han recibido. Es por ello que en este artículo analizaremos los factores que influyen en la exclusión del modelo del pensamiento crítico, así como también las ventajas de su implementación en el estudiante y en la sociedad. Además, se profundizará en las características, habilidades, actitudes que esto debe reunir para su desarrollo.

Palabras clave: Pensamiento crítico, desarrollo del pensamiento, profesores de literatura, disposición del profesor, inferencias

\section{Abstract}

Human beings have knowledge that they acquire throughout their lives. However, this knowledge can be developed differently by the models and approaches they have received.

That is why in this article we will analyze the factors that influence the exclusion of the critical thinking model, as well as the advantages of its implementation on students and in society. In addition, characteristics, skills, and attitudes that this implementation must gather for its development will be deepened.

Keywords: Critical thinking, thinking development, professor of literature, professor willingness, inference.

\section{Factores que influyen en el desarrollo del pensamiento crítico}

Algunos factores que influyen en el desarrollo del pensamiento crítico radican en el propio interés, motivación y práctica del docente quien es el que guía, facilita y evalúa el aprendizaje. En esta

1. Magister. Profesor regular de literatura del idioma Inglés. 
sección, analizaremos los componentes de cada factor, examinando los posibles obstáculos que impiden al docente de literatura ejecutarlos en clase.

En primer lugar consideraremos las anotaciones que hace Marciales (2003):

Existen una serie de factores que pueden estar afectando las posibilidades de desarrollo del pensamiento crítico. Uno de tales factores es el diseño de los currículos cual ha sido visto como determinante para que los profesores se centren en los contenidos fundamentalmente durante sus clases, más que en el desarrollo del pensamiento crítico ( $p$. 237-238).

A decir verdad, los profesores deben seguir lineamientos para unificar y cumplir con los estándares universitarios, es por ello que la necesidad de dicho cumplimiento los lleva a la realidad académica que consiste en seguir los contenidos de los programas previamente aprobados. Sin embargo, esto no quiere decir que al incluir recursos, técnicas metodológicas y actividades que desarrollen el pensamiento crítico hará que se modifiquen los contenidos del programa. Al contrario, cumpliendo con la estandarización curricular, también se cumple el desarrollo del pensamiento crítico no solo para ser cumplido por el profesor quien con su astucia innovará actividades para cumplir lo establecido en los programas, sino, para que se concrete en el estudiante, quien absorberá propiamente los contenidos que les beneficiará para las demás asignaturas a lo largo del semestre y las que tomará en el futuro.

El segundo factor tiene que ver con la orientación que los profesores hayan tenido en los comienzos de su carrera y la tendencia a la cual se dirigen. En este aspecto, Marciales (2003) señala que "los profesores reciben poca asesoría en relación con lo que puede ser considerado un "buen” pensamiento; es decir que, en general, no tienen claro qué es lo que se supone que deben ayudar a desarrollar en sus estudiantes" (p. 238). En teoría, esto podría ser la variable para una hipótesis. ¿Están los profesores suficientemente preparados para asumir el rol de agentes contribuyentes al pensamiento crítico? Si no lo están, tendremos profesores que ni siquiera asignarán tareas donde se desarrollen tales cometidos, y si lo están, el resultado debería ser obvio al cabo de un semestre, e inclusive al culminar la carrera. Aun mas, sería notorio en la sociedad cuando se les entrevista en la calle para que den su opinión acerca de alguna problemática o asunto concerniente a su país o al mundo y sus argumentos tengan fundamento y veracidad, evadiendo así al ridículo al que se somete ante el escrutinio de la comunidad.

Para profundizar al respecto, analizaremos lo que Sternberg (1987), citado por Marciales (2003) estableció:

Existen ocho falacias de los profesores respecto a la enseñanza y el aprendizaje, que inhiben la posibilidad de desarrollo del pensamiento crítico. 
1. Profesores que creen que no tienen nada que aprender de los estudiantes. En el campo del pensamiento crítico, el profesor es también un aprendiz que necesita ser receptivo a nuevas ideas.

2. Pensamiento crítico es solamente asunto del profesor. Así, el profesor debe pensar las respuestas y presentarlas suavemente, usando la mejor tecnología disponible para ello.

3. Existe el mejor programa para enseñar el pensamiento crítico. Al respecto, Sternberg estableció que no existe el mejor programa para esto, y los resultados dependen no solamente del programa sino también de las metas que se persiguen; asimismo, incide en ello el contexto o la cultura en la cual el pensamiento del aprendiz está situado.

4. La elección de un programa de pensamiento crítico está basado en un número binario de elecciones. Usualmente lo que puede resultar efectivo es la combinación de un amplio rango de aproximaciones.

5. Énfasis en la respuesta correcta, cuando lo que realmente es importante es el pensamiento detrás de la respuesta

6. La discusión es un medio para un fin. Sin embargo, quienes han ahondado en el tema consideran que el pensamiento crítico puede ser considerado un fin en sí mismo.

7. La noción de maestría en el aprendizaje: se espera que el estudiante logre un $90 \%$ de respuestas correctas en un $90 \%$ del tiempo establecido para esto. Usualmente el

pensamiento y la ejecución pueden ser mejorados más allá, existiendo condiciones para ello.

8. El papel de un curso sobre pensamiento crítico es enseñar pensamiento crítico Lo más probable es que la mayoría de los cursos no enfaticen aproximaciones que promuevan el "buen pensamiento", y que haya insuficientes oportunidades para los estudiantes para practicar análisis, crítica, síntesis y otros aspectos del pensamiento (p. 238-239).

Referente a la primera falacia, se puede añadir que así mismo como el doctor cree que no puede aprender del paciente, o el presidente de una nación de un ciudadano, así mismo pasa, en algunos casos entre profesor y estudiante. Hablamos de diferentes escenarios, pero con la misma convicción que da a lugar un pensamiento pobre abatido por el orgullo y desesperación que se atribuye a los pocos argumentos para debatirlo.

Al enfocarnos en la segunda falacia donde se piensa que el único responsable de desarrollar el pensamiento crítico es el profesor, se estaría culpando a este mismo de convertirlo en un pensamiento egoísta que se aísla en una realidad ensimismada y estéril. Así lo plantea Niño (2015), donde menciona que:

En ese camino didáctico, luego, se puede ver que el material utilizado para promover la interpretación de carácter monológico conceptual (dirige la interpretación hacia una sola forma de razonamiento) y este material, en realidad, termina desplazando el discurso polifónico y evaluativo del cuento ( $p$. 70).

Por otro lado en la tercera falacia, al decir que no hay el mejor programa para enseñar el pensamiento crítico, se lleva a la conclusión que no se debe seguir un solo patrón, sino un conjunto de herramientas que dan lugar al complemento del fin mismo. Así como no hay solamente un libro que incluya todos los cuentos infantiles del mundo o todos los poemas, de la misma manera trabaja 
el pensamiento crítico. El investigar constantemente actividades, teorías al respecto y demás, permitirá, en esa medida, al profesor a continuar, renovar, cambiar y actualizar su propio saber.

La cuarta falacia nos habla de que los profesores creen que la elección de un programa de pensamiento crítico está basada en un número binario de elecciones. En efecto, pueden haber programas que trabajen de par en par, no obstante en esta disciplina, como lo es el pensamiento crítico, funciona de manera más amplia; incluye una gama de habilidades cognoscitivas que al trabajarlas todas juntas se completa el raciocinio y por ser de tal naturaleza su efectividad, este toma tiempo en desarrollar.

En cuanto a la quinta falacia donde el profesor piensa que se debe hacer énfasis en la respuesta correcta, cuando lo que realmente es importante es el pensamiento detrás de la respuesta. Podemos ampliar que el pensamiento crítico no se trata de quien tiene la razón o si un argumento es correcto e incorrecto siempre y cuando este se enfoque en un argumento reflexivo con argumentos validados. Ciertamente, cuando desarrollamos el pensamiento crítico no hablamos de tener una respuesta correcta e incorrecta como cuando corregimos cierto y falso, escoger la mejor respuesta, llenar espacios o pareo. En estos últimos ejercicios, la memoria y la exactitud de los componentes juegan otro papel, no así cuando evaluamos un argumento basado en evidencias, reflexión, interpretación de un cuento, novela o poema. Además, en esta área de la ciencia, se incurre en el error de desatender o destruir sin indagar un argumento llevando al que lo propició, a la decepción o al ridículo. Así lo confirma Marciales (2003):

No atacar al argumentador sino por el contrario, criticar la posición reconstruyendo y evaluando los argumentos ofrecidos. Se trata de entender el argumento como un todo antes de romperlo y atacarlo en partes. Si no se puede entender es necesario explicar por qué y preguntar para mayor clarificación (p. 66).

Profundizando aún más en las respuestas o argumentos correctos o no, podemos enfatizar que algunas veces el profesor aspira a que el estudiante lo convenza de su argumento, sin considerar que detrás del argumento existe un esmero que contribuye a explicarlo. Así lo confirma Herrera (2018) "No es objeto de esta disciplina determinar si un argumento es o no persuasivo; si convence o no. Quien argumenta pretende aproximarse a la verdad de las cosas, sin considerar si ese esfuerzo tiene un fin persuasivo o no" ( p.18).

Al evaluar los contenidos nos lleva a otro contexto. Si el currículo nos indica los contenidos que deben ser evaluados y no tomamos en cuenta la reflexión, los profesores estarían evaluando solamente la comprensión de los mismos, mas no la reflexión que estas generen. Y muchas veces lo que esto provoca es que cuando se haga, hará que los estudiantes tomen un respiro de un currículo saturado (Marciales, 2003, p.212). De hecho, si los profesores se concientizaran, que al venir de un 
sistema tradicional donde la memoria jugaba un papel preponderante, permitieran a sus estudiantes desarrollar el pensamiento crítico se rompieran paradigmas que para algunos es difícil no seguir. Pero, la realidad en algunos casos es otra, se repiten las mismas prácticas tradicionales que al parecer son simples o fáciles para el que las utiliza.

En esta dirección, tomaremos otro punto de vista. Si el profesor incluyese actividades para desarrollar el pensamiento crítico, ¿estaría preparado para abordajes que van más allá de su conocimiento del tema. Yendo aún más lejos ¿tendría el tiempo para escuchar y evaluar los argumentos generados de la discusión de los temas de las lecturas por tener un gran número estudiantes en cada grupo? Recordemos que para una discusión en clase hay que escuchar a varios estudiantes ¿tendrán todos los estudiantes la oportunidad de dar su punto de vista?

Lo que si podemos asegurar es que el estudiante que no participe por falta de tiempo o falta de argumentos, tiene la capacidad para discernir, inferir y evaluar todos los puntos de vista de sus compañeros, lo que permitirá generar el suyo propio.

Seguidamente tenemos la sexta falacia, que señala que los profesores consideran que la discusión del pensamiento crítico es un medio para un fin, ya bien sea para atacar las malas prácticas del gobierno, el deterioro de los sistemas de salud o transporte u otros temas sensitivos o conflictivos. Si bien la discusión se presta para tales cometidos, el espacio sería propicio para generar el espacio para otros menesteres como por ejemplo, el desarrollo de los contenidos curriculares, siempre y cuando el profesor permita discusiones polifónicas, de lo contrario se podría confirmar que la discusión es para un fin específico del profesor.

Observemos lo que sucede en los cursos de literatura. Los análisis de algunos autores o de algunas fuentes de internet se prestan para aceptarlos como última evidencia. Es más, en algunas ocasiones se debe aceptar lo que piensa y dice el profesor, y es esto en lo que se debe basar el estudiante para escribir en sus asignaciones, y peor aún en sus pruebas. Aun más inadmisible, si sus ideas, punto de vista y argumentos son diferentes a las del profesor, muchas veces son tomados como incorrectos.

En esta particularidad Marciales (2003) establece humildad para no caer en dogmatismos, y audacia para superar muchos obstáculos, entre ellos, los que imponen aquellos que creen saber. Esta práctica conllevaría al hábito memorístico que se ve reflejado en las centros académicos donde la memoria es esencial, indiscutible e inapelable, y que a largo plazo no corre con buenos resultados por carecer de retentiva. Prueba de ello son las tablas de multiplicar que todavía en secundaria y nivel universitario, para irnos mas lejos, no son recordados. Y si seguimos enumerando, podemos sumarle las fechas nacionales históricas del país, que todavía quedan en el olvido.

Esta descripción la hace Niño (2015) al referirse a este tipo de método que utiliza el profesor: "no es más que solipcista, memorístico y monológico, privando al estudiante de su identidad, de su voz y su particular entender" (p. 26). De esta manera Marciales (2003) simplifica en pocas palabras que 
"los profesores invitan a los estudiantes a aceptar la experiencia de otros como válida, pues temen que esta sea dañada por los cuestionamientos. El proceso reflexivo puede conducir al abordaje de asuntos que van más allá del conocimiento del profesor" (p.212).

Dicho de otra manera, si los profesores creen verse amenazados por los cuestionamientos de los estudiantes por no tener la capacidad cognitiva, y no permitirlo en ninguna de sus clases, estas discusiones polifónicas no se ejecutarán. La séptima falacia hace mención que el pensamiento crítico es percibido por los profesores como la noción de maestría en el aprendizaje. El concepto que algunos profesores tienen es que mínimo, los estudiantes de primer y segundo año deben tener la capacidad de un egresado universitario, y además lograr tener un porcentaje alto en aciertos en sus argumentos, cuando la realidad es que para lograr el desarrollo del pensamiento crítico se necesita enfatizar en practicar habilidades para lograrlo como lo son la reflexión, inferencia y discusión, entre otras habilidades. Ya en la octava y última falacia hace pensar al profesor que el papel de un curso sobre pensamiento crítico es enseñar pensamiento crítico. A esta falacia podemos resaltar, que cuando se refiere a desarrollar el pensamiento crítico, no se está manifestando de enseñar pensamiento crítico como una corriente filosófica en los cursos de literatura, sino desarrollar sus características en los cursos de literatura. Si no se promueven sus características a lo largo de curso de literatura, no puede lograrse tal desarrollo como lo es practicar análisis, crítica, síntesis y otros aspectos del pensamiento. 


\section{Disposición del profesor}

En cuanto al desarrollo del pensamiento crítico por parte del profesor de literatura, hay que contemplar algunas observaciones. En primera instancia, en el proceso enseñanza-aprendizaje hay dos protagonistas principales que deberían ir en una sola dirección para lograr un fin determinado. Tanto el profesor como el estudiante juegan un papel preponderante para llevar a cabo los objetivos de los contendidos contemplados en el currículo, ambos se necesitan para progresar. El primero no es más importante que el último, y el último no es menos importante que el primero. El proceso de aprendizaje nunca termina y ambos se complementan y ajustan para el logro de sus necesidades y objetivos. Si el profesor está dispuesto a desarrollar el pensamiento crítico en sus clases de literatura, va a perseverar que se logre, utilizando sus habilidades y disposición, pese al tiempo (buscando alternativas) o número de estudiantes (solucionando problemas).

Esto lo ilustra muy claramente Ennis (1995) citado por Batista (2016):

El pensamiento crítico abarca un grupo de disposiciones: áreas básicas de pensamiento crítico, área de estrategias y área de tácticas que permiten desarrollarlo y utilizarlo. Así, el pensamiento crítico involucra disposiciones y habilidades cognitivas. En el sentido de identificar y enumerar las habilidades que permiten el desarrollo de este tipo de pensamiento, Ennis (1995), operacionalizó su concepción de pensamiento crítico y determinó cinco aéreas básicas de habilidades. Ellas son: clarificación elemental, soporte básico, inferencia y las estrategias y tácticas. Las habilidades que se desarrollan a partir de estas áreas, permiten al profesor convertirse en un investigador de su propia práctica pedagógica, en el sentido de buscar vías, métodos, alternativas y posibilidades para solucionar los problemas relacionados con la escuela de hoy, proporcionando apertura de criterios, justeza de ideas y cambio de paradigmas conservadores, en favor de un estudiante más acorde con su tiempo, creativo, crítico, desarrollado y sujeto activo de las transformaciones sociales (p. 13-14).

Si el profesor está dispuesto a desarrollar sus clases basadas en el pensamiento crítico, esto involucrará esfuerzo, sacrificio y compromiso con el estudiantado, y salir de un área de confort. Esta última fundamentada en los esquemas tradicionales donde se evalúa enunciados solipsistas, reincidiendo en los mismos métodos todos los años; métodos que los estudiantes consideran repetitivos, e inclusive algunas veces estereotipan a los profesores por utilizarlos. La autonomía lectora debería ser promovida por el profesor. Sin embargo esto dependerá de su grado de compromiso con el estudiantado. Estimular el interés o pasión a la lectura, satisfaciendo la 
preferencia de cada uno puede ser el primer comienzo. Si orientamos solamente los contenidos de las obras al género ficción, estaríamos limitando a los que prefieren no ficción a contenido que no les satisface. Igualmente, valorar el potencial, talento e inteligencia de los estudiantes para que publiquen obras, poemas y ensayos en foros sería de gran ayuda. De igual modo, apreciar sus comentarios y argumentos favorece ese estímulo a la lectura; puesto que, desafortunadamente desestimándolos no coadyuvaría al desarrollo, al contrario, los alejaría aún más de la lectura. "El profesorado debe alentar a los estudiantes para que lean más allá de los niveles más superficiales del texto y descubran los significados que estos niveles esconden" (lipman citado por Gómez, 2016, p.14). Lecturas complementarias para el desarrollo de sus habilidades lectoras deben venir del estudiante interesado en progresar en sus argumentos para que los mismos tengan validez y se puedan debatir en un ambiente de moción y reflexión. Es pues uno de los roles del profesor de literatura propiciar que los estudiantes desarrollen el pensamiento crítico a través de su autonomía lectora; no obstante sin su disposición este cometido no surtiría efecto.

Por eso es necesario que el profesor acompañe al estudiantado en ese proceso. Esta práctica no solo le producirá efectos a corto plazo en literatura, sino en las demás asignaturas, aun cuando en las demás no se esté desarrollando el pensamiento crítico. Concretamente este escenario está estrechamente ligado al crecimiento personal del docente quien se debe exigir a si mismo como agente innovador, investigador, comprometido, entusiasta, motivador, activo, entre otras características. De esta manera lo expresa Lipman citado por Gómez, (2016):

El pensamiento crítico y el crecimiento personal están relacionados. Por una parte, el pensamiento crítico necesita del crecimiento personal y a su vez hace que avance en forma positiva y viceversa. Para el desarrollo del pensamiento crítico es necesario que el docente genere ambiente crítico y es deber ser del docente universitario propiciar este ambiente. (p.251)

Pero, nuevamente, resaltamos lo mismo, si el profesor no tiene la disposición, interés ni habilidades para crear este ambiente, el desarrollo del pensamiento crítico no se generará.

Según Zúñiga (2012):

Hay algunas propuestas para desarrollar la capacidad analítica en los estudiantes para que sean autónomos en sus aprendizajes:

Olvidar el sistema de apuntes o de utilización básica de manuales.

$\square$ El profesor aclara conceptos y sirve de guía, pero deja que los estudiantes analicen y aprendan desde la comparación y acumulación de diversos referentes.

Fomentar el debate entre todos los estudiantes y el profesor. 
Los métodos para evaluar pueden ser trabajos que se relacionan con la evaluación continua y el examen escrito (no memorístico), es decir analítico, como por ejemplo; desarrollar un comentario de un tema (p.433).

Escrudiñemos cada uno de ellos:

Primero, olvidar el sistema de apuntes. Al eliminar este sistema el estudiante podría tener más comprensión cognitiva que estar preocupado en ver si se le quedó algo por escribir y que el profesor tomará en cuenta para un ejercicio sumativo. Al estar enfocado en la comprensión da oportunidad de emitir juicios que le permitan hacer preguntas en sitio para esclarecer dudas, da respuesta a la segunda propuesta donde el profesor aclara conceptos sirviendo de guía para futuras asignaciones.

Segundo, fomentar el debate entre todos los estudiantes y el profesor. Al permitir este espacio durante un análisis literario se logra adentrarse hasta comprender. Además, permite la preparación de argumentos que van a ser sometidos y los cuales involucra la participación del estudiantado quien tendrá que tomar su tiempo para investigar. "No se busca oponer un saber contra otro saber sino permitir el diálogo de dos o más experiencias, manteniendo el espacio abierto para que cada uno encuentre su propia inquietud" (Marciales, 2003, p.104). Esto no quiere decir que al haber una discusión entre el colectivo se intenta fomentar una pelea en un ring de boxeo o juzgar quién es mejor y quién es peor, o quién ganó y quién perdió. Al contrario, al estar preparados con argumentos permite a todas las partes involucradas en el desarrollo de un tema, crear un ambiente de razonamiento donde la verdad sobresalga o donde se comprueben o refuten los planteamientos. Y por último, la tercera propuesta relacionado a la evaluación, se acentúa en la evaluación continua y examen no memorístico donde se evidencia el resultado del aprendizaje cognitivo el cual no será complejo para el estudiante si este mantuvo una discusión y búsqueda activa referente al tema. Esta evaluación dará un descanso o alivio a los métodos utilizados hasta el momento. Por consiguiente, así lo recalca Marciales (2003) Muchos estudiantes toman el tiempo destinado a la reflexión como un medio para "tomar un respiro" de un currículo saturado (p.212). Es pues, una propuesta saludable dejar que los estudiantes experimenten el placer de reflexionar y dar opiniones. Al principio, los resultados no darán frutos a corto plazo, puesto que el ejercicio de ejecutarlos donde involucra pensar más allá de sus posibilidades e investigar continuamente, comparar una idea con la otra, hacer algo a lo que no se estaba acostumbrado es complejo y toma tiempo. Sin embargo, la paciencia, la continuidad y la responsabilidad contribuirán a desarrollarlo. Tomaremos ahora, el siguiente cuadro para explicar los enfoques tradicionales y las características que catalogan a la lectura como formación. Esto en miras a ilustrar posteriormente ejemplos concretos como sugerencias para la lectura como formación y como aviso a los enfoques tradicionales que no permiten avanzar en el desarrollo del pensamiento crítico. 


\begin{tabular}{|l|l|}
\hline ENFOQUES TRADICIONALES & LA LECTURA COMO FORMACIÓN \\
\hline $\begin{array}{l}\text { La lectura se considera como un proceso de } \\
\text { transferencia de significado que requiere que } \\
\text { los lectores extraigan el significado de la letra } \\
\text { impresa. }\end{array}$ & $\begin{array}{l}\text { La lectura es un proceso de construcción y de } \\
\text { producción de sentido entre el lector, el texto, } \\
\text { los otros significativos y el contexto. }\end{array}$ \\
\hline $\begin{array}{l}\text { Los lectores son consumidores pasivos de los } \\
\text { textos y significados de otros. }\end{array}$ & $\begin{array}{l}\text { Los lectores se transforman en la interacción } \\
\text { con el texto. }\end{array}$ \\
\hline $\begin{array}{l}\text { El profesor enseña técnicas para extraer los } \\
\text { significados del texto. }\end{array}$ & $\begin{array}{l}\text { El profesor comparte su experiencia de } \\
\text { relación con el texto. }\end{array}$ \\
\hline $\begin{array}{l}\text { Los estudiantes son capacitados para } \\
\text { describir los significados que se consideran } \\
\text { apropiados. }\end{array}$ & $\begin{array}{l}\text { Los estudiantes son acompañados para que } \\
\text { puedan encontrar sus propias inquietudes. }\end{array}$ \\
\hline $\begin{array}{l}\text { Las situaciones de aprendizaje son } \\
\text { individuales, recurriendo al trabajo en grupo } \\
\text { solamente como medio para lograr la } \\
\text { conformidad con los significados definidos } \\
\text { por una autoridad ajena al lector. }\end{array}$ & $\begin{array}{l}\text { El compartir experiencias se considera } \\
\text { comprensión. }\end{array}$ \\
\hline $\begin{array}{l}\text { La enseñanza se orienta hacia técnicas de } \\
\text { aplicación universal. }\end{array}$ & $\begin{array}{l}\text { La enseñanza se orienta a la valoración de las } \\
\text { finalidades perseguidas por el lector y la } \\
\text { escucha de sus propias comprensiones. }\end{array}$ \\
\hline
\end{tabular}

Cuadro 1. Adaptado de Cainney (1.992) citado por Marciales (2003), p. 112

Enseñar literatura, permite al profesor una gama de actividades que enriquezcan el proceso enseñanza-aprendizaje, sin embargo, hay una parte del profesorado que solo utiliza el enfoque tradicional, el otro lo utiliza la lectura como formación para desarrollar el pensamiento crítico.

Es por ello que se enumerará una lista de ejemplos para cada uno de los enfoques.

\begin{tabular}{|c|c|}
\hline $\begin{array}{l}\text { ENFOQUES TRADICIONALES } \\
\text { (Actividades para estudiantes) }\end{array}$ & $\begin{array}{l}\text { LA LECTURA COMO FORMACIÓN } \\
\text { (Actividades para estudiantes) }\end{array}$ \\
\hline $\begin{array}{l}\text { Ejemplo: -¿Qué es fábula? } \\
\text { ¿Quiénes son los protagonistas?, } \\
\text { - ¿Qué vio Caperucita en el bosque? ¿Qué } \\
\text { significa caperucita? }\end{array}$ & $\begin{array}{l}\text { Ejemplo:- ¿Qué se destaca en esta fábula? } \\
\text { ¿De qué manera influye esta fábula en tu } \\
\text { vida personal? } \\
\text { - ¿Cómo se aplica la palabra malicia en este }\end{array}$ \\
\hline
\end{tabular}




\begin{tabular}{|c|c|}
\hline & $\begin{array}{l}\text { cuento? ¿Qué enunciados son } \\
\text { incompatibles con su realidad, De ejemplos } \\
\text { comparándolos? }\end{array}$ \\
\hline $\begin{array}{l}\text { Ejemplo: -Lean el cuento Cenicientas y } \\
\text { hagan un resumen. } \\
\text {-Conteste las siguientes preguntas. - } \\
\text { ¿Cuántas hermanastras tenía Cenicientas? } \\
\text { ¿A qué hora Cenicientas tenía que irse del } \\
\text { palacio? }\end{array}$ & $\begin{array}{l}\text { Ejemplo: -Una vez leído el cuento de } \\
\text { Cenicientas, dos estudiantes tomarás el papel } \\
\text { de las hermanastras de Cenicientas, otro de } \\
\text { la madrastra, otro de la hada madrina, otro } \\
\text { del príncipe, y así sucesivamente. El resto } \\
\text { de los estudiantes interrogará a los } \\
\text { protagonistas. Por ejemplo: ¿Por qué te } \\
\text { portaste tan mal con Cenicientas? ¿Príncipe, } \\
\text { qué te cautivó de Cenicientas? } \\
\text {-Crear un debate (incluir grupo a favor y otro } \\
\text { en contra) y un panel de discusión (incluir } \\
\text { protagonistas, psicólogos, entre otros. }\end{array}$ \\
\hline $\begin{array}{l}\text { Ejemplo: Subrayen las palabras nuevas para } \\
\text { usted. }\end{array}$ & $\begin{array}{l}\text { Ejemplo: Una vez leído El Patito Feo, el } \\
\text { profesor da un testimonio de lo que le pasó } \\
\text { cuando era pequeña. Ella sufría de bullying } \\
\text { y veinte años después se encontró a uno de } \\
\text { sus acosadores y este no la reconocía, y ella } \\
\text { lo abordó y le dijo yo soy el patito feo, y el } \\
\text { chico quería una cita con ella, a lo que ella } \\
\text { rechazó. } \\
\text { Luego, el profesor da oportunidad para que } \\
\text { lo estudiantes opinen. }\end{array}$ \\
\hline $\begin{array}{l}\text { Ejemplo: Hagan oraciones con las palabras } \\
\text { del vocabulario tomado de la fábula o } \\
\text { cuento. }\end{array}$ & $\begin{array}{l}\text { Ejemplo: El profesor analiza los recursos } \\
\text { retóricos y literarios de un cuento, novela o } \\
\text { poema con ejemplos. Luego el profesor } \\
\text { facilita la comprensión mediante el método } \\
\text { socrático. }\end{array}$ \\
\hline $\begin{array}{l}\text { Ejemplo: Agrupar de } 5 \text { a } 6 \text { miembros para } \\
\text { contestar preguntas } \\
\text {-Dibujar una escena del cuento, novela, } \\
\text { fabula o poema en } 30 \text { minutos. }\end{array}$ & $\begin{array}{l}\text { Ejemplo: -Agrupar en equipos de } 5 \text { o } 6 \text { para } \\
\text { que cada uno comente una experiencia } \\
\text { referente al bullying (lo pueden dramatizar o } \\
\text { escribir). } \\
\text {-Escribir una historia donde contemplen } \\
\text { todas las experiencias tomando en cuenta por } \\
\text { lo menos } 4 \text { figuras retoricas o literarias. }\end{array}$ \\
\hline $\begin{array}{l}\text { Ejemplo: Contestar cierto y falso, escoger la } \\
\text { mejor respuesta, completar un pareo. }\end{array}$ & $\begin{array}{l}\text { Ejemplo: -Escribir un ensayo/poema/cuento } \\
\text { considerando } 2 \text { figuras literarias o retoricas. }\end{array}$ \\
\hline
\end{tabular}

Cuadro 2. Elaborado por el autor de este articulo

Es pues, disposición del profesor asumir su papel como agente del pensamiento crítico y formar estudiantes con tales criterios o permanecer en su zona de confort siguiendo las mismas prácticas que a lo mejor sus profesores seguían.

\section{Ventajas al desarrollar el pensamiento crítico}

Se considera el provecho que se le saca al desarrollar el pensamiento crítico en los estudiantes. Zarzar (2016) nos resalta que "el desarrollar la capacidad para el pensamiento crítico nos puede 
ayudar de muchas formas en nuestra vida personal, social, familiar, escolar y laboral " (p.76). Esto tiene un beneficio para la sociedad al contar con ciudadanos capaces de escuchar antes de hablar, pensar antes de actuar y tomar decisiones frente a un conflicto. Estas habilidades no se pueden obtener de mentes acostumbradas a memorizar y al comportamiento robótico de asignaciones que no permiten que el pensamiento se desarrolle por sí solo.

Al favorecer el desarrollo del pensamiento crítico en los estudiantes, se estaría cumpliendo con una responsabilidad social.

Esto también lo sostiene Duque, (2012) cuando dice que al desarrollarse la comprensión inferencial contribuye al desenvolvimiento en la vida. "La comprensión inferencial no solo permite leer lo implícito en el texto para lograr un esclarecimiento de su estructura profunda, sino que propicia el desarrollo de competencias para desenvolverse en la vida y en diversas áreas de conocimiento" (p.4).

Daremos un ejemplo más real. Al desarrollarse la inferencia en las clases, el estudiante pusiera en marcha sus habilidades cognitivas a la hora de resolver un problema midiendo las ventajas y desventajas, buscando soluciones y pensaría las consecuencias de sus actos antes de actuar o hablar. Además, coadyuvaría al desarrollo de sus otras clases, aun cuando en estas no se estén desarrollando las habilidades del pensamiento crítico.

"Si es posible favorecer el desarrollo del pensamiento crítico en nuestros jóvenes universitarios y futuros profesionales, y no lo estamos haciendo, ¿estaremos fallando en nuestro compromiso ético como formadores?” (Marciales, 2003, p.84) Esta es la pregunta que todos los profesores deberían formularse antes de planear, durante el proceso enseñanza-aprendizaje y después de evaluar a los estudiantes. Ilustremos cada uno de ellos. Antes de planear, el profesor debe saber los contenidos y los objetivos del programa, pero además tener presente que los contenidos pueden desarrollarse de manera reflexiva, incluyendo análisis, investigación y participación activa promoviendo el pensamiento crítico y creativo del estudiante. Para ello necesitará romper paradigmas tradicionales y salir del área de confort al que a lo mejor estaba acostumbrado como por ejemplo los que ya hemos mencionado (ejercicios de escoger la mejor respuesta, llenar espacio, entre otras). Durante el proceso enseñanza-aprendizaje, el profesor debe contar que modificar técnicas de aprendizaje, añadir contenido asociado a los señalados en el programa, considerar oportunidades a los estudiantes en el desarrollo de un argumento, recapacitar en alguna aseveración intransigente serán de apoyo para desarrollar el pensamiento crítico. Y finalmente, después de evaluar a los estudiantes, el profesor debe analizar el porcentaje de fracaso. Si su libreta indica que hay un alto índice de fracaso, varios factores pueden estar afectando. Solo el profesor podría indagar. O es el profesor quien no está cumpliendo su compromiso como formador o es el estudiante quien no le está llegando el conocimiento del contenido por algunas circunstancias. 


\section{Habilidades y actitudes para desarrollar el pensamiento crítico}

A lo largo de este artículo se ha estado señalando las habilidades del pensamiento crítico, y a la vez se han estado mencionando algunas. Es por ello, que dedicamos en la siguiente sección las habilidades y lo que cada una de ellas representa para el desarrollo del mismo. El pensamiento crítico se conforma de grandes perspectivas que algunos autores han explicado y que al tomarlas en cuenta conforman las habilidades del pensamiento crítico. Tomaremos en primera instancia las que el autor Batista (2016) nos comparte: “interpretación, el análisis, la evaluación, la inferencia, la explicación y la autorregulación" (p.24). Por un lado al desarrollar la interpretación de una obra literaria, estaríamos cumpliendo con más habilidades que conllevan otras habilidades. Para poder interpretar una obra literaria tendríamos que concentrarnos para entenderla, sintetizarla, e identificar elementos literarios, para luego poder explicarlo. Por otro lado el análisis, a su vez también abarca otras capacidades como lo son: destacar lo más relevante, estudiar a fondo el propósito del mismo, valorar lo captado y concluir con un argumento sostenido con evidencias. Para ilustrar este último aspecto Herrero, (2018) nos dice que "para llegar a una conclusión cuando la cuestión es más compleja (y no se trata de optar), es preciso tener razones, evidencias, debidamente respaldadas, que nos permitan sostener aquello por lo que nos decantamos" (p.17). En cuanto a la evaluación, podemos mencionar que reconocer el alcance de la obra literaria, así como también el juicio que se emane del mismo, son ejercicios mentales que el pensador crítico debe considerar. Ahondaremos en la inferencia como parte de las habilidades del desarrollo del pensamiento crítico. Esta definición la explican Belinchon, Riviere \& lgoa 2000; Graesser \&Wiemer'Hastings, 1999; Jurado, Bustamante \& Pérez, 1998, citados por Duque (2012) “las inferencias son definidas como representaciones mentales que el lector/oyente construye o añade al comprender el texto, a partir de las aplicaciones de sus propios conocimientos a las indicaciones explícitas en el mensaje”( $p .4)$

Si las inferencias son representaciones mentales, esto quiere decir que antes que se representen en nuestra mente, tienen que ser procesadas mediante el mecanismo de comprensión. O sea, que lograr inferir, no puede ser el primer o único paso para desarrollar el pensamiento crítico, este necesita de otras capacidades para que se lleve a cabo. Los profesores no pueden asignar una tarea a los estudiantes los primeros días del semestre donde se deba inferir, si antes no se ha cumplido con los pasos de interpretación, análisis y evaluación de una obra literaria.

Un ejemplo concreto en el área de literatura lo sugiere Lipman citado por Gómez (2016):

Puede ser de gran ayuda que el profesorado proponga al alumnado que encuentre ejemplos de pensamiento creativo en un capítulo de una novela que se esté discutiendo. O bien, que 
pregunte qué tipos de cuidado muestran los personajes en un episodio. Estas preguntas hacen que los estudiantes hagan una lectura profunda del texto porque deben ser capaces de inferir (p.13).

Supongamos que dos ejemplos creativos de la fábula 'El León y el Ratón' sean: Hacer la dinámica en el salón para experimentar que tan fuerte tenían que ser los dientes del ratón para romper la malla que atrapaba al león. Y el segundo ejemplo sería medir el tiempo para ver qué tiempo había tomado el ratón para romper la malla. En cuanto al tipo de cuidado que muestran los personajes, podemos mencionar el cuidado que tuvo que haber tomado el ratón para que no lo sorprendieran los cazadores.

Por otro lado, es preciso añadir que las inferencias son parte importante a la hora de desarrollar el pensamiento crítico. Inferir es un proceso simple para algunos, pero complejo para otros. Su desarrollo viene con la práctica de una serie de saberes que conducen a una conclusión o un conjunto de demostraciones mentales que el estudiante se ha estado formulando para llegar a una decisión.

El pensamiento ocurre cuando existe un esfuerzo orientado a la coordinación de inferencias para llegar a una conclusión que, da respuesta a un problema, o que obedece a un proceso de toma de decisiones, o a la justificación de un planteamiento, entre otros (Marciales, 2003, p.76).

Por ejemplo, si la interrogante por parte del profesor es ¿cuál es el enunciado que mejor resume el cuento ? y explique por qué, podría claramente evidenciar parte de lo enunciado.

Retomando las dos últimas habilidades, pero no menos importantes, que mencionaba Faria (2005) las cuales son: la explicación y la autorregulación, podemos indicar que por un lado la explicación debe determinar como el estudiante enfoca la obra literaria. Analizándola podría precisar una introducción, desarrollarla y luego concluirla. Y con respecto a la autorregulación, podemos concluir que es una secuencia de las demás habilidades usadas como los son: el análisis, la confirmación y validación de evidencias, así como también de la concienciación de la deducción e inferencia, siendo responsable del resultado de su propio argumento.

Otro autor que nos explica otras habilidades que son importante al desarrollar el pensamiento crítico es Zúñiga, (2012). Él señala que algunas habilidades que se relacionan con el pensamiento crítico son: comparar, resumir, observar, clasificar, interpretar, criticar, descubrir suposiciones, colectar y organizar datos y tomar decisiones (p.435). Traduzcamos estas habilidades a destrezas cognoscitivas que el estudiante ha utilizado a lo largo de la primaria y secundaria para llevar a cabo sus faenas diarias, pero esta vez utilizándolas todas juntas para llegar a un fin. ¿Cuántas veces un estudiante no ha tenido que comparar dos cuentos o dos objetos y observar sus características para 
luego hacer un resumen? ¿Cuántas veces no ha tenido que interpretado las palabras de un vocabulario para después clasificarlas? ¿Y cuántas veces no ha tenido que descubrir suposiciones coleccionando y organizando datos para tomar decisiones? Así que resumiendo lo que Zúñiga nos comparte, podemos decir que a pesar de que hay algunas habilidades que los estudiantes han empleado a lo largo de su vida escolar, todas se relacionan con el pensamiento crítico, solo que el profesor es responsable de facilitar su implementación para desarrollar el pensamiento crítico.

A su vez, también aporta Zarzar (2016) acerca de las habilidades del pensamiento crítico y reitera lo que ya algunos autores arriba mencionados nos han compartido, y además agrega actitudes y conocimientos que el pensador crítico posee:

\section{LAS CARACTERÍSTICAS DE UN PENSADOR CRÍTICO.}

Habilidades: Analítico, observador, investigador, para la lectura, para razonar, creativo. Inferencia, explicación, interpreta, evaluación, autorregulación. Actitudes: Realista, objetivo, reflexivo, espontaneidad, innovador.

Conocimientos: generales, específicos, criterios, cultura general (p. 58).

Si completamos las habilidades, con las actitudes y los conocimientos que nos muestra Zarzar y los otros autores, estaríamos hablando de una serie de características que nos llevan a la determinación que para desarrollar el pensamiento crítico se necesitan la gran mayoría y en algunos casos todos, pero también es pertinente adicionar que el docente pieza fundamental en el proceso.

Algunos elementos adicionales que se deben tener presente para desarrollar el pensamiento crítico y que tiene que ver con la actitud son contexto, estrategia y motivación.

Contexto para comprender y poder opinar, al tener un panorama general de los hechos el estudiante podrá emanar juicios. Ejemplo: Si la asignación fue leer una novela, y el estudiante la lee, esto quiere decir que ya tiene un panorama de la novela para poder emitir de manera razonada y coherente a las preguntas que le diera el profesor. El estudiante sería capaz de responder a: qué, cómo, quién(es), cuánto(s), dónde, por qué, cuando, etc.

* Estrategia para crear un plan y cumplir con los objetivos, al contar con bases, argumentos podrá debatir y emitir conclusiones. Ejemplo: El estudiante que sí leyó la novela asignada por el profesor, podrá opinar, pero a la vez, oirá los comentarios de los demás y lo ayudaría a despejar dudas y ampliar su horizonte de comprensión. Esas son algunas de las estrategias empleadas para lograr una comprensión total. el contexto también permite mantener una visión razonada y coherente con la situación.

* Motivación para avanzar en el proceso, con este se facilita y aligera el desarrollo del conocimiento y se hace más llevadero. Ejemplo: Si el estudiante al leer la novela no le 
genera ningún tipo de interés, su conexión con el texto será vagamente comprendido. Es por esta razón que el profesor será responsable en la elección de la obra literaria tomando en consideración los gustos, preferencias y actualidad mundial para agradar a los estudiantes y estos no incurran en el aburrimiento. Romper paradigmas también tiene que ver con el cambio de actitud del profesor; además, tiene que ver con detener su práctica repetitiva de asignar las mismas novelas y cuentos que acostumbraba a incluir en su planeamiento desde que inició su labor docente.

El autor Sibauste et al., (2001) citado por Marciales (2003) lo explica en el siguiente enunciado:

Tres elementos han de ser tenidos en cuenta al hablar de pensamiento crítico: contexto, estrategias y motivaciones. El primero, es el contexto del sujeto que le exige responder de manera razonada y coherente con la situación. Las estrategias, son el conjunto de procedimientos de los cuales dispone el sujeto para operar sobre los conocimientos que posee y aquellos nuevos. Finalmente, las motivaciones hacen referencia al vínculo que establece el sujeto con el conocimiento, ese vínculo afectivo que mueve su curiosidad e invita al desarrollo de una actitud positiva frente al conocer ( $p .60)$.

Hemos visto, pues, como un todo complementa el desarrollo del pensamiento crítico. El pensamiento no es un conocimiento que actúa solo, sino un método basado en razonamiento que echa a un lado la memoria por un momento y se dedica a reflexionar, interpretar, inferir, entre otras prácticas ya mencionadas anteriormente. El pensamiento crítico no consiste en algunas habilidades, sino de la integración de la comunicación, investigación, lectura, escucha y razonamiento:

No considera que el desarrollo del pensamiento crítico consista en seleccionar y pulir unas cuantas habilidades que se creen necesarias, sino que se trata de empezar a tratar con los amplios campos de la comunicación, de la investigación, de la lectura, de la escucha, del habla, de la escritura y del razonamiento, y se ha de cultivar cualquier habilidad que provea un dominio de este tipo de procesos intelectuales (Marciales, 2003, p.59).

Por otra parte no se puede desmentir a la premisa que el pensamiento crítico es una habilidad intelectual compleja. Y mucho más si esta no se ha desarrollado lo suficiente. En un semestre podremos encontrar que algunos cursos solo se limiten a memorizar, mientras que en otros se enfocan en reflexionar. Lidiar con esta realidad no es sencillo porque una avanza a la reflexión y la otra a la memoria. Una ya es costumbre, la otra empieza a emerger.

Cuando se dice que el pensador crítico requiere ciertas actitudes como cuestionar, dudar, afán por la verdad y sensibilidad al contexto, se debe tomar en cuenta que el pensamiento deja de ser pasivo para convertirse en activo y que esta forma de pensamiento abre amplios cuestionamientos donde la duda juega el papel primordial de futuras decisiones y conclusiones que resultarán ser nuevos paradigmas. Para ampliar en este sentido, se puede tomar como ejemplo el momento que toma el 
profesor para discutir el tema: 'Los niños no deben hablar con extraños' como único tema de una novela, cuento, fabula o poema en particular. El profesor brinda su opinión para respaldar su punto de vista e ilustra con ejemplos las consecuencias. A este punto, si se permite la participación activa del estudiante, puede surgir el cometario 'Hablar con extraños con sensatez' debería ser el tema por tales razones, y otro estudiante puede refutar diciendo 'siempre y cuando tenga sensatez y no sea tan inmaduro' alegando que no todos los niños son maduros y diferencian quién aparenta tener buenas intenciones y quién no. 


\section{Conclusión}

El profundizar acerca del desarrollo del pensamiento crítico nos permite revelar que el papel que juega el profesor en el proceso enseñanza-aprendizaje es preponderante, ya que sin su disposición no se pudiera lograr tal desarrollo. El docente es el responsable de fomentar la participación reflexiva en los estudiantes quien no es más que un receptor que debe acatarse a los enfoques que el profesor dictamine. El pensamiento crítico queda expresado como un conjunto de habilidades, actitudes y conocimientos que se ejecutan para lograr la reflexión e inferencia. Al ser utilizadas en conjunto paso a paso ayudaría de gran manera al desarrollo del pensamiento. Por otro lado, es relevante mencionar que los factores que influyen en que el docente desarrolle sus clases basados en pensamiento crítico están estrictamente relacionados a su disponibilidad, deseo, actitud, formación, compromiso y dedicación que este dedique a su profesión. Además, podemos concluir que este artículo se puede usar para concienciar no solo a los profesores de literatura, sino de todas las áreas del saber, ya pudieran meditar de su labor docente como innovadores, investigadores, comprometidos, motivadores y activos para optimizar y adaptar lo necesario para un mejor aprovechamiento de los estudiantes.

En general, el desarrollo del pensamiento crítico en las clases contribuiría enormemente a la sociedad quien carece enormemente de personas reflexivas que tomen decisiones contundentes y generen solución a los problemas sociales y económicos.

\section{Referencias}

Batista, João. (2016). Desarrollo de habilidades para contribuir al pensamiento crítico de los estudiantes en la Educación de Jóvenes y Adultos (EJA), a través del proceso de enseñanza aprendizaje de la historia de Brasil .Tesis de Doctorado. La Habana : Editorial Universitaria.

Duque, Claudia . (2012). Inferencias sobre un texto narrativo en contextos de interacción en la educación inicial. Universitas Psychologica. Vol. 11(2). 4

Herrero, Julio César. (2018). Elementos del pensamiento crítico (2a. ed.). Alcalá de Henares: Marcial Pons Ediciones Jurídicas y Sociales.

Lipman, Matthew. (2016). El lugar del pensamiento en la educación . Barcelona: Ediciones Octaedro.

Marciales Gloria Patricia. (2003). Pensamiento crítico: diferencias en estudiantes universitarios en el tipo de creencias, estrategias e inferencias en la lectura crítica de textos. Tesis de Doctorado. Madrid: Universidad Complutense de Madrid S.A. 
Niño Rojas, C. A. (2015). Representaciones sociales desde el docente y sus implicaciones en la didáctica literaria en el género de cuento: hacia una comprensión de los procesos de formación del pensamiento crítico en estudiantes de secundaria. Ibagué, Colombia: Sello Editorial Universidad del Tolima.

Zarzar, Carlos. (2016). Métodos y pensamientos críticos . México: Grupo Editorial Patria.

Zúñiga, María. (2012). Los estudiantes universitarios del siglo xxi en México: de la pasividad a la autonomía y al pensamiento crítico. Revista Teoría de la Educación. Educación y Cultura en La Sociedad de la Información. Vol. 13. № 2. 